\title{
Bovine miR-146a regulates inflammatory cytokines of bovine mammary epithelial cells via targeting the TRAF6 gene
}

\author{
Xing-Ping Wang, ${ }^{*} \dagger^{1,2}$ Zhuo-Ma Luoreng, ${ }^{*}{ }^{1}$ Lin-Sen Zan, ${ }^{* 2}$ Feng Li, $\dagger$ and Na Li† \\ ${ }^{*}$ College of Animal Science and Technology, National Beef Cattle Improvement Center, Northwest A\&F University, Yangling Shaanxi, \\ 712100 , China \\ †Key Laboratory of Zoology in Hunan Higher Education, College of Life and Environmental Science, Hunan University of Arts and Science, \\ Changde Hunan, 415000, China
}

\begin{abstract}
It has been reported previously that bovine miR-146a (bta-miR-146a) is significantly differentially expressed in mammary glands infected with mastitis, compared with healthy udders. This suggests that bta-miR-146a plays an important role in the regulation of mammary inflammation. However, the specifics of this function have yet to be elucidated. Bovine mammary epithelial cells (bMEC) represent the first line of defense against pathogens and have important roles in initiating and regulating inflammatory responses and innate immunity during infection. In this study, a double luciferase reporter assay was used to confirm that bta-miR-146a directly targets the $3^{\prime}$ UTR of the tumor-necrosis factor receptor-associated factor $6(T R A F 6)$ gene. To elucidate the role of bta-miR-146a in innate immune responses, either a mimic or inhibitor of bta-miR-146a was transfected into bMEC stimulated with lipopolysaccharide, which activates the innate immune response through the toll-like receptor (TLR) 4/nuclear factor (NF)- $\kappa \mathrm{B}$ signaling pathway. Forty-eight hours posttransfection, quantitative real-time PCR and Western blots were used to detect the expressions of the related genes and proteins, respectively. An ELISA was used to measure the quantity of inflammatory factors in culture supernatants. The results showed that bta-miR-146a significantly inhibits both mRNA and protein expression levels of bovine TRAF6, and ultimately suppresses downstream expression of NF- $\kappa \mathrm{B}$ mRNA and protein. As a result, production of NF- $\kappa \mathrm{B}$-dependent inflammatory mediators such as tumor necrosis factor $\alpha$, IL-6, and IL-8 are suppressed following lipopolysaccharide stimulation of bMEC. Thus, we concluded that btamiR-146a acts as a negative feedback regulator of
\end{abstract}

Received January 23, 2017.

Accepted May 15, 2017.

${ }^{1}$ These authors contributed equally to this work.

${ }^{2}$ Corresponding authors: huaswxp@hotmail.com and zanlinsen@163. com bovine inflammation and innate immunity through downregulation of the TLR4/TRAF6/NF- $\kappa \mathrm{B}$ pathway. This study presents a potential regulatory mechanism of bta-miR-146a on immune responses in bovine mammary infection and may provide a potential therapeutic target for mastitis.

Key words: miR-146a, bovine mammary epithelial cell, inflammation, innate immunity

\section{INTRODUCTION}

Bovine mastitis, an inflammation of the mammary gland caused by bacterial infection, is a highly prevalent and costly disease of dairy cattle (Seegers et al., 2003). Infection of the bovine mammary gland by pathogens modulates inflammatory cytokines in bovine mammary epithelial cells (bMEC), which recruit white blood cells to the sites of infection (Sordillo et al., 1997; Rainard and Riollet, 2006). During infection of bovine mammary glands, bMEC serve as the first line of defense against invading pathogens, and play a major role in the initiation of immune responses (GriesbeckZilch et al., 2008). These cells are capable of detecting pathogen-associated molecular patterns (PAMP) of invading pathogens through toll-like receptors (TLR), activating the nuclear factor (NF)- $\kappa \mathrm{B}$ pathway and the associated innate immune responses (Huang et al., 2012; Wellnitz and Bruckmaier, 2012; Gilbert et al., 2013; Gunther et al., 2016). Lipopolysaccharide, the major component of the outer membrane of gramnegative bacteria, is a common trigger of inflammation. It has been widely used to model bacterial interactions with mammalian cells, including bMEC (Wellnitz and Kerr, 2004; Strandberg et al., 2005; Gilbert et al., 2013; Wang et al., 2016a). Previous studies have indicated that stimulation of bMEC with LPS elicited a marked increase in the gene expression of inflammatory cytokines and chemokines, such as tumor necrosis factor (TNF) $\alpha$, IL-6, and IL-8 (Strandberg et al., 2005; Gilbert et al., 2013; Wang et al., 2016a).

MicroRNA (miRNA) are endogenous, noncoding, small RNA molecules that regulate innate and adaptive 
immunity by directly binding to the $3^{\prime}$ untranslated region (UTR) of the target mRNA (O'Connell et al., 2010; Lawless et al., 2014). Previously, we reported that the expression of bovine miR-146a (bta-miR-146a) was significantly increased in cases of bovine mastitis caused by Staphylococcus aureus, Escherichia coli, or clinical mixed bacteria (Wang et al., 2016b). However, Naeem et al. (2012) reported the expression of btamiR-146a was not altered in response to Streptococcus uberis infection of mammary glands in vivo. These referenced studies suggest that the expression of btamiR-146a varies in response to mammary tissue infection with the specific invading pathogen. Interestingly, it has been reported that induction of human miR-146a (has-miR-146a) is NF- $\kappa \mathrm{B}$-dependent and regulates innate immune responses by targeting the tumornecrosis factor receptor-associated factor 6 (TRAF6) and IL-1 receptor-associated kinase (IRAK1) genes (Taganov et al., 2006). Notably, the sequences of miR146a, TRAF6, and IRAK1 between bovine and human exhibit a high degree of nucleotide identity. However, whether or not bta-miR-146a has the same function as has-miR-146a requires further study. Moreover, the role and the detailed mechanisms of bta-miR-146a functions in response to infection of bMEC remain unclear. In this study, we sought to identify the target genes of bta-miR-146a and elucidate its potential mechanism on inflammatory responses in LPS stimulated-bMEC.

\section{MATERIALS AND METHODS}

\section{Cell Culture}

The HEK293 cells were cultured in high-glucose Dubecco's modified Eagle medium (HyClone Laboratories, South Logan, UT) supplemented with $10 \%$ fetal bovine serum (Clark Bioscience, Richmond, VA). The bMEC were cultured in Dubecco's modified Eagle medium/ F12 containing 10\% exosome-depleted fetal bovine serum (System Biosciences, Mountain View, CA). All cells were incubated in a humidified atmosphere containing $5 \% \mathrm{CO}_{2}$ at $37^{\circ} \mathrm{C}$. For cytokine analysis, bMEC were incubated with $50 \mathrm{ng} / \mu \mathrm{L}$ of LPS for $3 \mathrm{~h}$ (Strandberg et al., 2005), which is capable of inducing an inflammatory response in bMEC, and to an extent, the bMEC model can reflect the in vivo response of mastitis.

\section{miRNA Mimics and Inhibitors}

Mimics of miR-146a/b (dsRNA oligonucleotides), mismatched negative control mimics (NC_mimic), and miR-146a/b inhibitor (single-stranded oligonucleotides), mismatched negative control inhibitor (NC_ inhibitor), each labeled with $\mathrm{Cy} 3$, were synthesized by
GenePharma Co., Ltd. (Shanghai, China). Mimics and inhibitors were used to assess the effects of overexpression and inhibition of miR-146a/b on its activity in HEK293 cells or bMEC, respectively.

\section{Target Prediction Analysis}

Bioinformatics-based target prediction analysis was performed using TargetScan 7.0 (http://www. targetscan.org/vert_71/) and miRBase (http:// microrna.sanger.ac.uk/).

\section{3' UTR Luciferase Reporter Assays}

Total RNA was extracted from bMEC using the Trizol reagent (Invitrogen, Carlsbad, CA) and was used to synthesize first strand cDNA using a PrimeScript II 1st Strand cDNA Synthesis Kit (Takara Biotechnology Dalian Co., Ltd., Dalian, China). Double-strand cDNA fragments of $3^{\prime}$ UTR of the potential target gene containing the putative miRNA binding site were amplified by RT-PCR. Site-specific mutated forms of the $3^{\prime}$ UTR were created using overlap extension PCR (primers presented in Supplemental Table S1; https:// doi.org/10.3168/jds.2017-12630). The double-strand cDNA fragment of the $3^{\prime}$ UTR and associated mutants were digested and cloned into the XhoI-NotI sites of the psiCHECK-2 vector, containing both renilla and firefly luciferase reporter genes (Promega Corp.), respectively. The recombinant plasmid was extracted with an EndoFree Plasmid Mini Kit (Omega Bio-tek, Norcross, GA) and constructs were verified by DNA sequencing.

The human embryonic kidney (HEK) 293 cells were plated at a density of $1.0 \times 10^{5}$ cells/well in 24-well plates. Twenty-four hours later, HEK293 cells were co-transfected with plasmids $(0.5 \mu \mathrm{g})$ or mimic (or NC_mimic; $0.5 \mu \mathrm{g}$, the final work concentration 75 $\mathrm{n} M$ ) using $2 \mu \mathrm{L}$ of X-tremeGENE HP DNA Transfection Reagent (Roche, Penzberg, Germany) following the manufacturer's protocol (https://pim-eservices. roche.com/LifeScience/Document/e36981cf-e722-e411ed82-00215a9b0ba8). At $48 \mathrm{~h}$ posttransfection, renilla luciferase activity, normalized to firefly luciferase activity, was detected using the Dual-Luciferase Reporter Assay System (Promega, Madison, WI) according to the manufacturer's instructions (https://cn.promega. $\mathrm{com} /$ resources/protocols/technical-manuals/0/dualluciferase-reporter-assay-system-protocol/).

\section{Transfection of bMEC}

Six-well plates were seeded with bMEC at 50 to $60 \%$ confluence. The cells were then transfected with mimic, NC_mimic, inhibitor, or NC_inhibitor (each 
labeled with $\mathrm{Cy} 3$ ) using the X-tremeGENE HP DNA Transfection Reagent (Roche, Germany) according to the manufacturer's instructions (https://pim-eservices. roche.com/LifeScience/Document/e36981cf-e722-e411ed82-00215a9b0ba8). Briefly, X-tremeGENE HP DNA Transfection Reagent $(4 \mu \mathrm{L})$ was diluted in $100 \mu \mathrm{L}$ of Opti-MEM (Invitrogen) with mimic, NC_mimic, inhibitor, or NC_inhibitor to yield final concentrations of 75 , 75,150 , and $150 \mathrm{n} M$, respectively, with gentle agitation. The mixture was incubated at room temperature for 20 min, then added to the bMEC. Transfection efficiency was assessed by fluorescence microscopy (Supplemental Figure S1; https://doi.org/10.3168/jds.2017-12630).

\section{RNA Isolation and Quantitative Real-Time PCR}

All quantitative real-time PCR (qPCR) experiments were designed and conducted in compliance with the MIQE guidelines (Bustin et al., 2009), the details of which, including PCR validation, are provided in Supplemental File S1 and Supplemental Figure S2 (https://doi.org/10.3168/jds.2017-12630). Briefly, total RNA and miRNA were extracted from the transfected bMEC using the Trizol reagent (Invitrogen) and the miRcute miRNA isolation kit (Tiangen Biotech Co., Ltd. Beijing, China), respectively. The concentrations and quality of miRNA and mRNA were measured using a BioSpec-nano Micro-volume UV-Visible Spectrophotometer (Shimadzu Scientific Instruments, Shimadzu, Japan). Quantitative PCR of miRNA and mRNA were performed using the miRcute miRNA qPCR Detection Kit with SYBR Green chemistry (Tiangen Biotech Co., Ltd.) and the SYBR Premix Ex Taq II kit (TaKaRa, Biotechnology Co. Ltd., Dalian, China), respectively, according to the manufacturer's instructions (http:// www.takarabiomed.com.cn/DownLoad/RR820A.pdf). Both a no-template control and no-reverse transcriptase control were included in all plates to confirm that amplification was not due to contamination or primer dimers. All reactions were performed using the ABI 7500 RT-PCR system (Applied Biosystems, Waltham, MA). The primers used in the study are listed in Supplemental Table S2 (https://doi.org/10.3168/ jds.2017-12630). The qPCR amplification efficiencies of the primer sets ranged from 100.16 to $104.80 \%$ with an $\mathrm{R}^{2}>0.99$ (Supplemental Figure S2; https://doi. org/10.3168/jds.2017-12630).

The relative expression quantification (fold changes) were calculated using the $2^{-\Delta \Delta \mathrm{Ct}}$ method (Livak and Schmittgen, 2001). Cel-miR-39 was used as a reference gene to normalize the expression of the detected miRNA. The geometric mean of the 3 reference genes ( $\beta$-actin, 18s rRNA, and UXT), which have been validated as reference genes of qPCR in the LPS-stimulated bMEC (Bougarn et al., 2011), was used to normalize the expression of mRNA, according to the method previously described by Vandesompele et al. (2002).

\section{Western Blot Analysis}

Western blotting was performed to detect expression of proteins related to miR-146a in bMEC. Control or transfected cells were lysed in RIPA buffer (Sangon Biotech Co. Ltd., Shanghai, China) containing 1\% phenylmethane sulfonyl fluoride. Total protein concentrations were quantified using the Bicinchoninic Acid Protein Assay kit (Sangon Biotech Co. Ltd.).

Equal quantities of total protein $(25 \mu \mathrm{g})$ from each sample were fractionated by $12 \%$ SDS-PAGE and transferred to nitrocellulose protran membranes (GE Healthcare/Amersham, Pittsburgh, PA). Membranes were blocked with $5 \%$ nonfat milk, and then probed with an appropriate dilution of primary antibody at $4^{\circ} \mathrm{C}$ overnight. All of the primary and the secondary antibodies, including TRAF6 (1:500, catalog no. sc-7221), NF-кB (1:500, catalog no. sc-8414), $\beta$-actin (1:500, catalog no. sc-47778), goat anti-mouse IgG-HRP (1:5,000, catalog no. sc-2005), and goat anti-rabbit IgG-HRP (1:5,000, catalog no. sc-2030), were purchased from Santa Cruz Biotechnology Inc. (Dallas, TX). Bands were visualized using ECL Substrates (Millipore, Billerica, MA). $\beta$-Actin was used as the loading control for normalization. Western blot images were analyzed using ImageJ software (http://imagej.nih.gov/ij/).

\section{ELISA for Cytokines}

Cell culture supernatants from mimic- or inhibitortransfected and LPS-stimulated bMEC were collected and stored at $-80^{\circ} \mathrm{C}$. Secretion of the cytokines IL-6, TNFo, and IL-8 in bMEC culture supernatants were measured by ELISA using the relative ELISA kits (Shanghai Enzyme-linked Biotechnology Co., Ltd., Shanghai, China) according to the manufacturer's instructions (http://en.mlbio.cn/).

\section{Statistical Analysis}

The expression levels of mRNA and protein were assayed in triplicate. All data are expressed as mean \pm standard deviation. Statistical analyses were performed using SPSS 18.0 (SPSS Inc., Chicago, IL). Statistical significance in the data between treatment groups (mimic or inhibitor transfected) and control groups (mimic negative control or inhibitor negative control transfected) was determined by the Student's $t$-test. 
$P$-values of $<0.05$ were considered to be statistically significant.

\section{RESULTS}

\section{Bta-miR-146a Directly Targets the TRAF6 Gene}

Previously, we reported that the expression of btamiR-146a and bta-miR-146b in mammary gland tissue infected with $E$. coli and $S$. aureus, or mixed clinical bacteria were significantly upregulated in comparison to healthy cows (Wang et al., 2016b). This suggests that bta-miR-146a and bta-miR-146b participate in the inflammatory process in bovine mammary glands. Thus, we conducted a more in-depth exploration of the target genes of bta-miR-146a and bta-miR-146b influencing bovine immune responses in this study.

Using the online miRNA databases (TargetScan 7.0 and miRBase), we predicted that the $3^{\prime}$ UTR of the TRAF6 gene contains both bta-miR-146a and btamiR-146b target sequences (Figure 1A). Interestingly, this was also reported to be a target of miR-146a in humans (Taganov et al., 2006).

To further investigate if the predicted binding sites of bta-miR-146a/b in the $3^{\prime}$ UTR of TRAF6 are responsible for the regulation, we cloned the $3^{\prime}$ UTR of TRAF6 (named TRAF6-wt) to the psiCHECK-2 vector (CHECK2_3'UTR-TRAF6-wt), and co-transfected CHECK2_3'UTR-TRAF6-wt and bta-miR-146a mimics (or negative control) into HEK293 cells. It was observed that in HEK293 cells co-transfected with CHECK2_3'UTR-TRAF6-wt and mimic exhibited a significant decrease in the relative luciferase activity compared with control groups (co-transfected with CHECK2_3'UTR-TRAF6-wt and NC_mimic; $P<$ 0.001; Figure 1B). Moreover, when the TRAF6 $3^{\prime}$ UTR was mutated (TRAF6-mut), it was found that bta-miR-146a-mediated repression of luciferase activity was abolished (Figure 1B). These results indicated that bta-miR-146a directly targets $3^{\prime}$ UTR of TRAF6 gene, leading to an inhibition of luciferase activity.

\section{Bta-miR-146b Does Not Directly Target the TRAF6 Gene}

As described above, the $3^{\prime}$ UTR reporter assay was performed, this time using bta-miR-146b. The results showed that the relative luciferase activities of HEK293 cells transfected with bta-miR-146b and CHECK2_3'UTR-TRAF6-wt were not significantly different compared with control groups $(P>0.05)$. Therefore, it is not believed that bta-miR-146b directly targets the TRAF6 gene (Figure 1C).

\section{Bta-miR-146a/b Does Not Directly Target the IRAK1 Gene}

The $3^{\prime}$ UTR of bovine IRAK1 containing the 3 predicted binding sites of bta-miR-146a and bta-miR-146b (Figure 2A) were obtained. It has been previously reported that IRAK1_site\#1 and IRAK1_site\#2 had also been identified as human miR-146a targets (Taganov et al., 2006), and IRAK1_site\#3 is the target of mouse miR-146a (Jiang et al., 2014). Primers for IRAK1-wt (Supplemental Table S1; https://doi.org/10.3168/ jds.2017-12630) were used to amplify the $3^{\prime}$ UTR of bovine IRAK1 containing the 3 target sites mentioned above for 3' UTR luciferase reporter assays. Although human and bovine miR-146a/b, and their putative target sequences of IRAK1, are highly homologous, the current luciferase reporter assays (Figure 2B) indicated that bovine IRAK1 is not a target gene of either btamiR-146a or bta-miR-146b.

\section{Bta-miR-146a Suppresses TRAF6 and NF-KB Expression in bMEC}

To explore the possible roles and associated molecular mechanism of bta-miR-146a in bMEC, we set out to determine whether bta-miR-146a regulates the posttranscriptional expression levels of TRAF6 and downstream $N F-\kappa B$ in bMEC. The qPCR analysis indicated that the bta-miR-146a mimic significantly increased the levels of mature bta-miR-146a (Figure 3A), and the bta-miR-146a inhibitor significantly decreased the level of bta-miR-146a in bMEC (Figure 3B). We then detected mRNA and protein expression of TRAF6 and $\mathrm{NF}-\kappa \mathrm{B}$ in bMEC at $48 \mathrm{~h}$ posttransfection with either bta-miR-146a mimics, inhibitor, or scrambled controls. The results showed that overexpression of bta-miR-146a in bMEC significantly reduced the levels of TRAF 6 and $\mathrm{NF}-\kappa \mathrm{B}$ at both mRNA and protein level in comparison with negative controls (NC_mimic) group $(P<0.01$; Figure $3 \mathrm{C}$ and $3 \mathrm{D}$ ). Conversely, downregulation of bta-miR-146a resulted in a significant increase in both $\mathrm{mRNA}$ and protein expression of TRAF 6 and NF- $\mathrm{KB}$ $(P<0.01$; Figure $3 \mathrm{E}$ and $3 \mathrm{~F})$. These results indicate that bta-miR-146a inhibits TRAF6 and $N F-\kappa B$ expression at the posttranscriptional level.

\section{Bta-miR-146a Negatively Modulates Expression and Secretion of TNFa, IL-6, and IL-8 in LPS-Stimulated bMEC}

TRAF6 and NF- $\kappa \mathrm{B}$ are involved in innate immune responses to infection. To investigate whether bta-miR146 a regulates the production of the pro-inflammatory 
cytokines TNFo, IL-6, and the chemokine IL-8, which are key mediators in the animal inflammatory response, we transfected bMEC with bta-miR-146a mimic or inhibitor. Twenty-four hours posttransfection, bMEC were stimulated with $50 \mathrm{ng} / \mu \mathrm{L}$ of LPS. After $3 \mathrm{~h}$, mRNA expression and secretion of TNFo, IL-6, and IL-8 were determined by qPCR and ELISA, respec- tively. As shown in Figure 4, the bta-miR-146a mimic significantly decreased the mRNA expression and production of TNF $\alpha$, IL-6, and IL-8 compared with the control group (Figure 4A, 4C, and $4 \mathrm{E}$ ). In contrast, the bta-miR-146a inhibitor significantly increased expression of the cytokines (Figure $4 \mathrm{~B}, 4 \mathrm{D}$, and $4 \mathrm{~F}$ ). These data indicate that bta-miR-146a suppresses mRNA
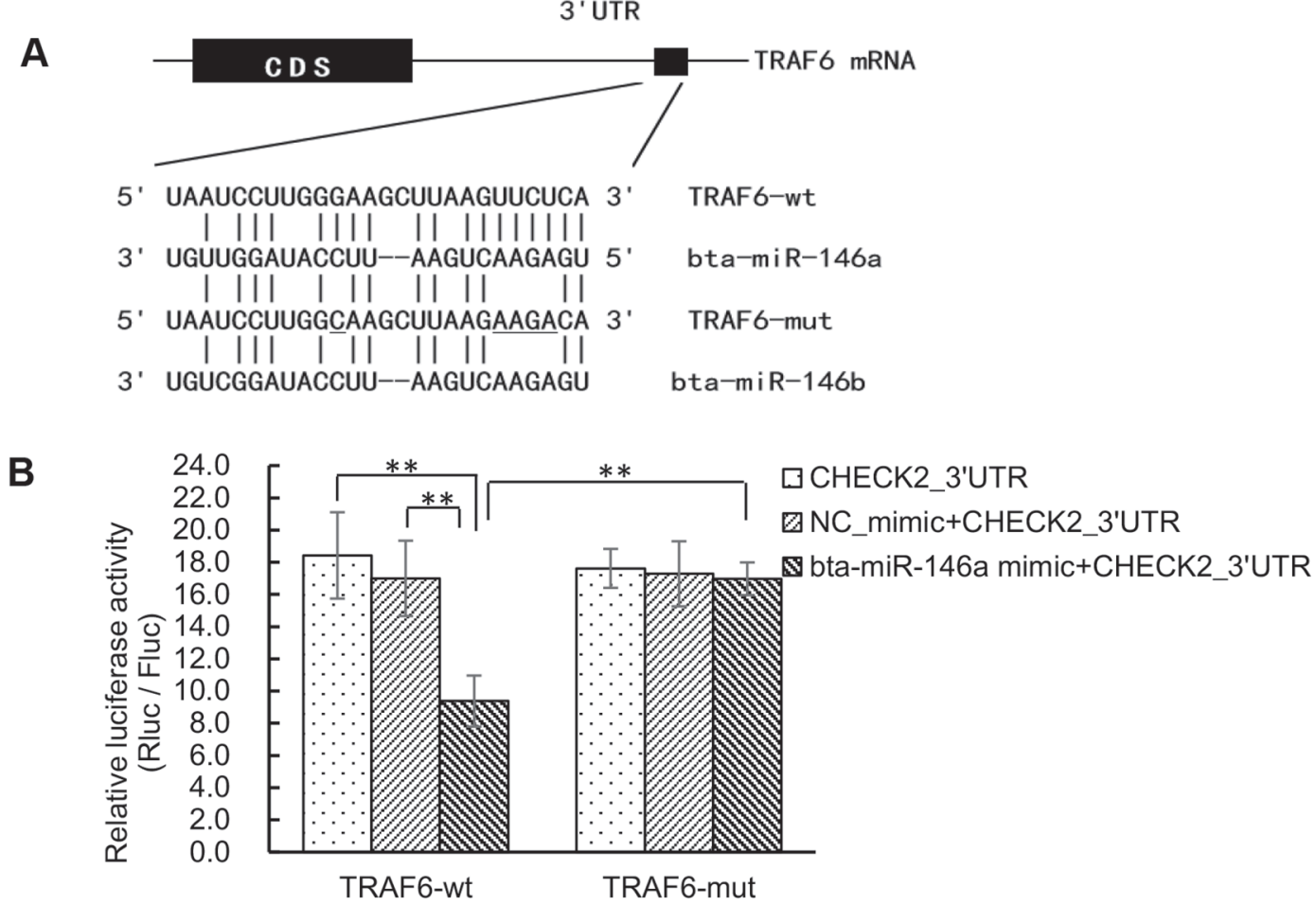

C

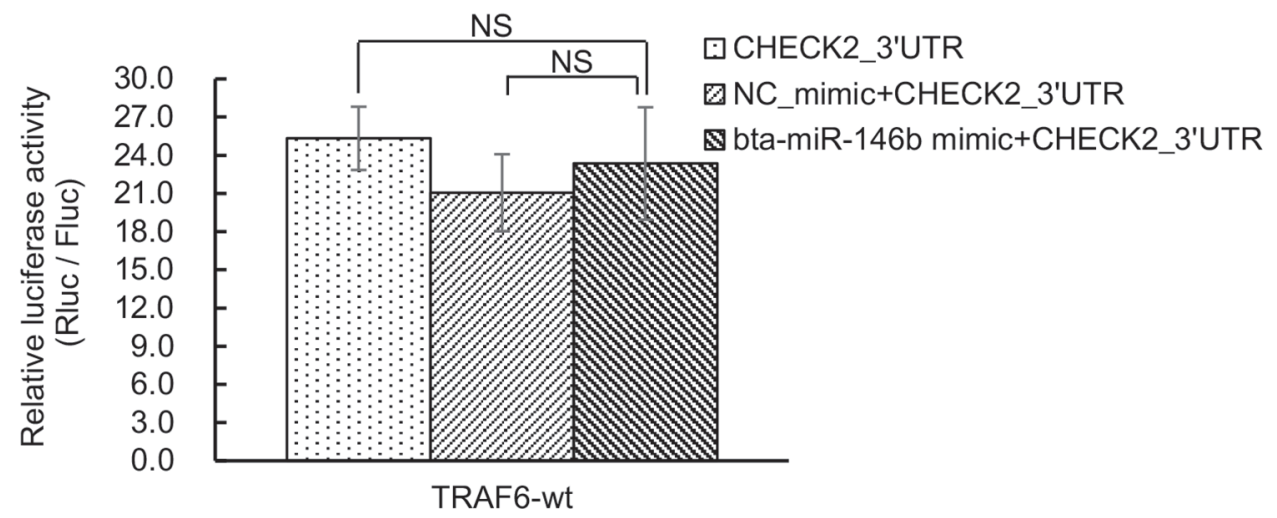

Figure 1. Bovine miR-146a (bta-miR-146a) directly targets the tumor-necrosis factor receptor-associated factor 6 (TRAF6) gene by binding to its $3^{\prime}$ untranslated region (UTR). (A) The predicted bta-miR-146a/b binding sites within $3^{\prime}$ UTR of TRAF6 and the experimentally introduced mutations are shown. (B) Verification of the bta-miR-146a target sites in the $3^{\prime}$ UTR of TRAF6 by dual-luciferase reporter assay. (C) Bta-miR-146b does not directly target the TRAF6 gene. This was confirmed through the 3' UTR dual-luciferase reporter assay. Renilla luciferase activity was normalized to the activity of firefly luciferase in the same recombination vector. Results are mean \pm SD. ${ }^{* *} P<0.001$; NS: $P>0.05$, as determined by paired 2-tailed Student's $t$-tests. CDS = coding sequence; NC_mimic = negative control, compared with bta-miR-146a/b mimics; TRAF6-wt $=$ wild type of target sites in TRAF6; TRAF6-mut $=$ site-directed mutagenesis of TRAF6; CHECK2_ $3^{\prime} \mathrm{UTR}=$ dual luciferase report recombinant vector of TRAF6-wt-3' UTR or TRAF6-mut-3' UTR and psi-CHECK-2; Rluc/Fluc = Renilla luciferase/firefly luciferase. 


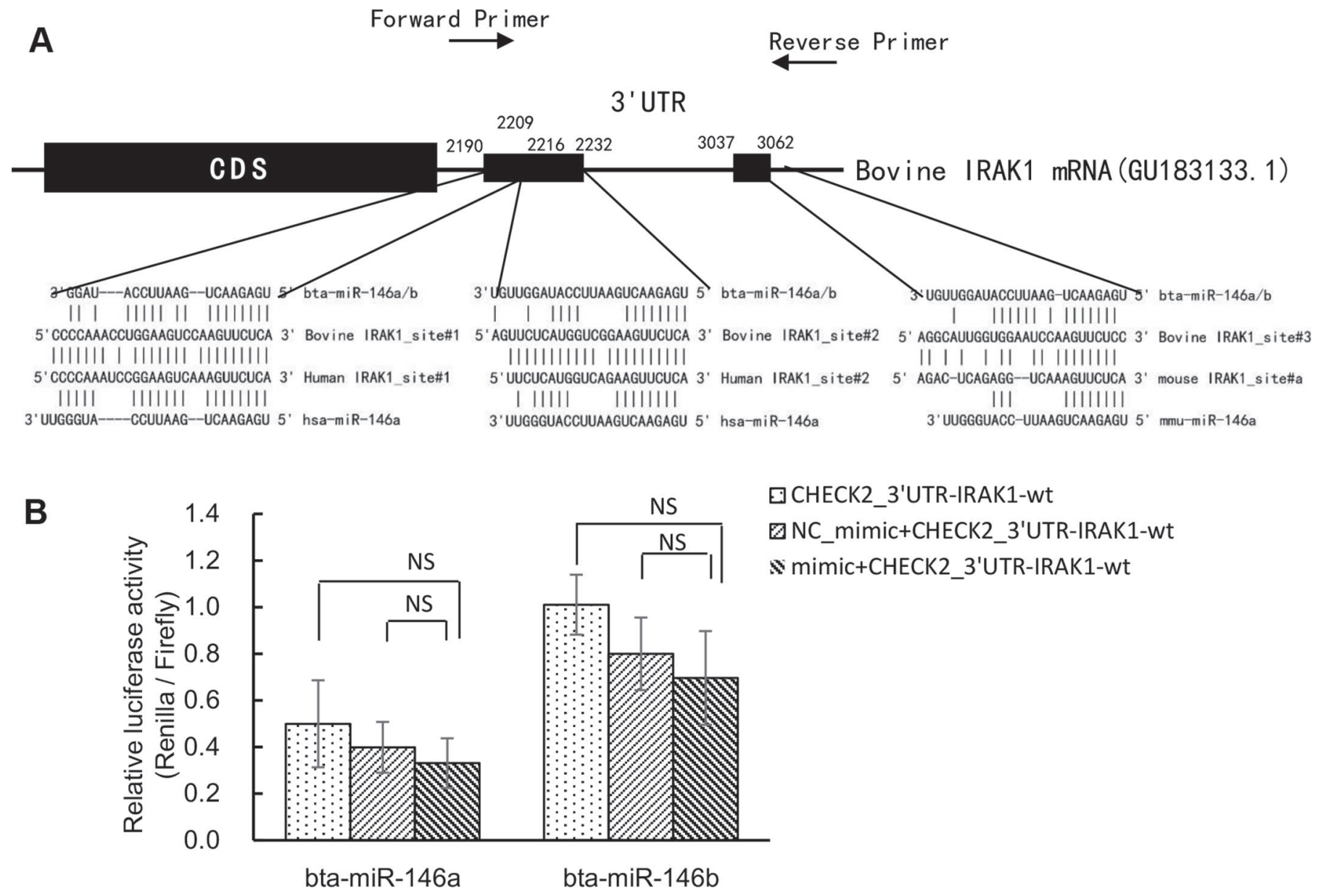

Figure 2. The IL-1 receptor-associated kinase (IRAK1) gene is not directly targeted by bovine miR-146a (bta-miR-146a) and bta-miR-146b. Panel (A) shows the sequence alignments of miR-146a/b and its putative targets in the $3^{\prime}$ untranslated region (UTR) of IRAK1 compared with the counterparts of human and mouse, which have been identified as targets of miR-146a/b in human (has-miR-146a/b) or mouse (mmu-miR146a). (B) Human embryonic kidney (HEK) 293 cells were transiently co-transfected with CHECK2_IRAK1-3UTR dual luciferase reporter recombinant vector, and bta-miR-146a (or bta-miR-146b) mimic or NC_mimic. Renilla luciferase activity was normalized to firefly luciferase activity in the same vector. Results are mean \pm SD. NS: $P>0.05 ;$ NC_mimic = negative control, compared with bta-miR-146a/b mimics; CHECK2_3' UTR-IRAK1-wt = IRAK1-3UTR-wt and psiCHECK-2 dual luciferase reporter recombinant vector.

expression and ultimately secretion of TNF $\alpha$, IL-6, and IL-8 in bMEC. Taken together, this suggests that btamiR-146a is a potential regulator of inflammation in the bovine mammary gland.

\section{DISCUSSION}

As novel, posttranscriptional modulators of gene expression, miRNA were recently found to be important regulators of mammalian immune responses (Taganov et al., 2006; Lawless et al., 2014; Park et al., 2015). In the present study, we provide evidence suggesting that bta-miR-146a regulates inflammation in bMEC by directly targeting the TRAF6 gene.

In humans and mice, miR-146a has been shown to be a key gene involved in host immunity. Studies have dem- onstrated that expression of miR-146a is significantly increased in infected human or mouse tissues (Gao et al., 2015; Dai et al., 2016), LPS-induced cells (Taganov et al., 2006), human monocytes and macrophages (Zeng et al., 2013), RAW264.7 cells (Li et al., 2013), dendritic cells (Park et al., 2015), and keratinocytes (Meisgen et al., 2014) during inflammatory responses. In agreement with these findings, our previous work found that expression of bta-miR-146a was increased significantly in mammary glands challenged with $S$. aureus or $E$. coli compared with the glands of healthy cows (Wang et al., 2016b). However, Naeem et al. (2012) reported that the expression of bta-miR-146a did not change in highdose $S$. uberis-infected mammary tissue, as compared with the expression in the mammary glands of healthy cows. The results of the above studies indicated that 
A

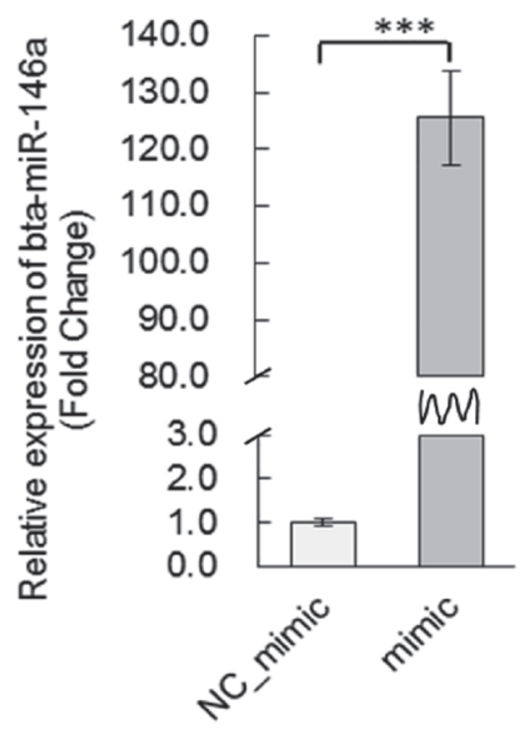

C

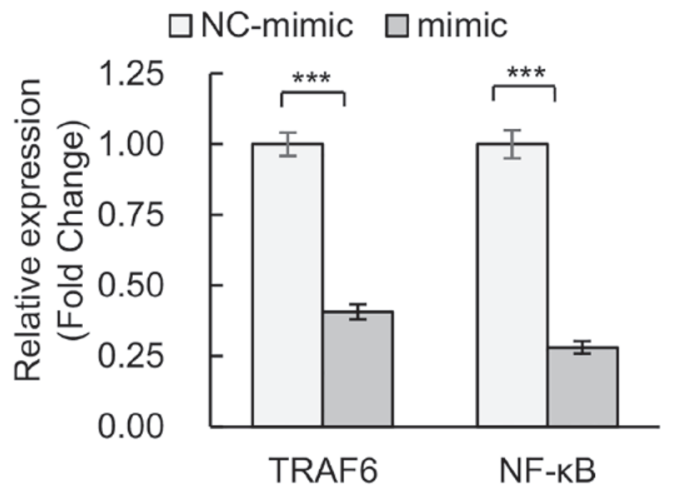

E

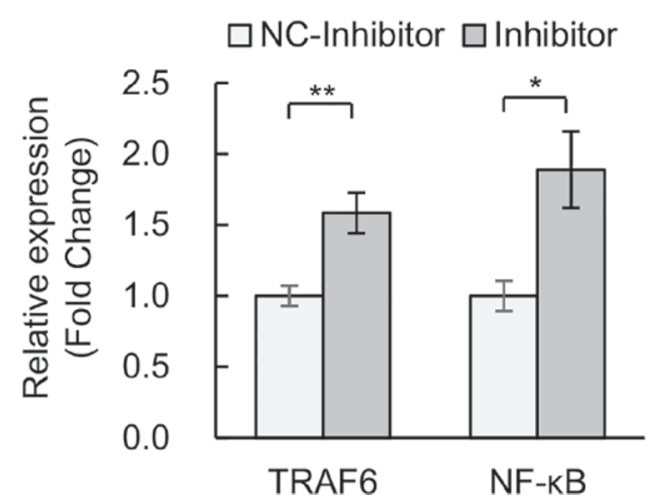

B

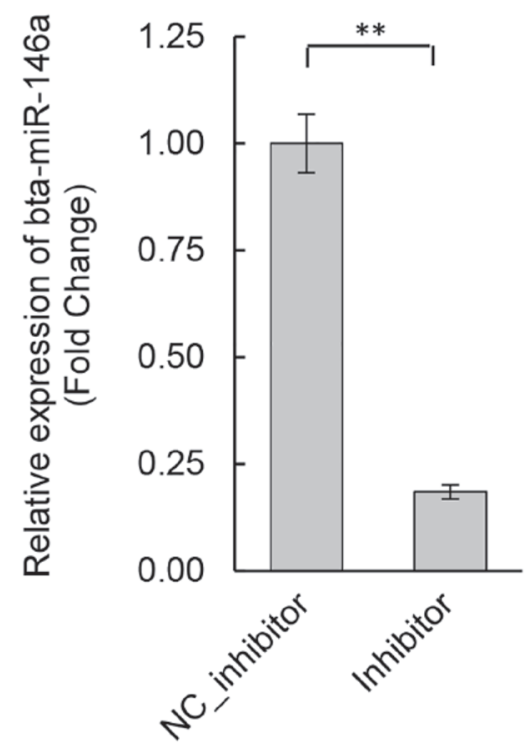

D

TRAF6

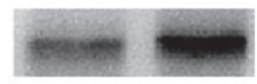

NF-KB

$\beta$-Actin
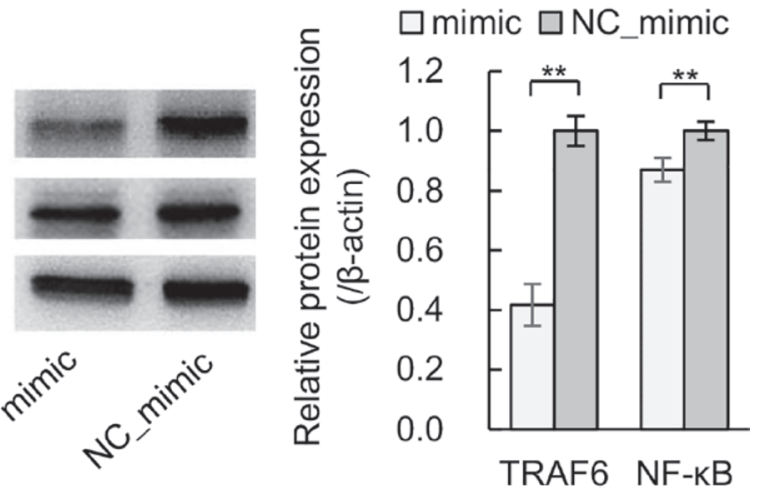

F

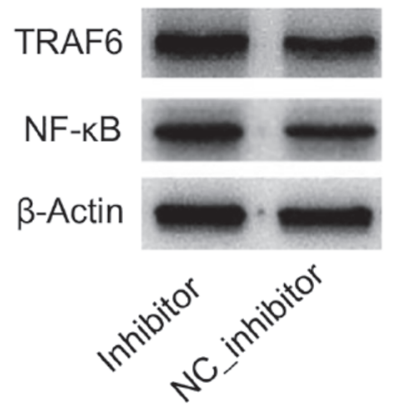

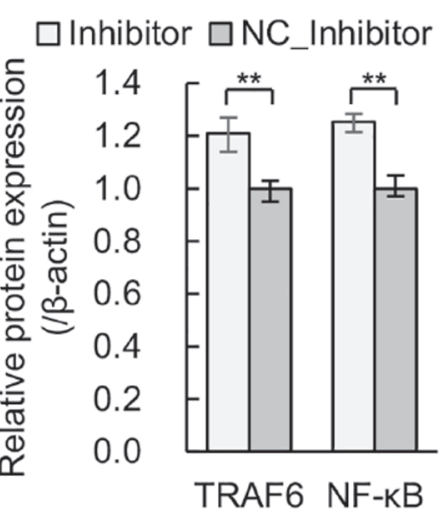

Figure 3. Bovine miR-146a (bta-miR-146a) suppresses the expression of the tumor-necrosis factor receptor-associated factor 6 (TRAF6) and nuclear factor (NF)- $\kappa \mathrm{B}$ in bovine mammary epithelial cells (bMEC). The bMEC were transfected with bta-miR-146a mimic or negative control mimic (NC_mimic), or either inhibitor or negative control inhibitor (NC_inhibitor). At $48 \mathrm{~h}$ posttransfection, quantitative real-time PCR (qPCR) and Western blot were used to measure the expression levels of genes and protein, respectively. (A) The transfection efficiency of bta-miR-146a mimic as measured by qPCR. (B) The transfection efficiency of bta-miR-146a inhibitor as measured by qPCR. (C) qPCR analysis of TRAF6 and $N F-\kappa B$ in bMEC transfected with bta-miR-146a mimic. (D) Western blot analysis of TRAF6 and NF- $\kappa$ B in bMEC transfected with bta-miR-146a mimic. (E) qPCR analysis of TRAF6 and NF- $\kappa B$ in bMEC transfected with bta-miR-146a inhibitor. (F) Western blot analysis of TRAF6 and NF- $\mathrm{KB}$ in bMEC transfected with bta-miR-146a inhibitor. Western blot images were analyzed using the ImageJ software (http://imagej.nih.gov/ij/), and densitometry quantification of Western blots were normalized to $\beta$-actin. Data are presented as means \pm SD. Statistically significant differences are indicated: ${ }^{*} P<0.05$, ${ }^{*} P<0.01$, ${ }^{* * *} P<0.001$. All experiments were repeated 3 times. 
A

$$
\text { TNFa (qPCR) }
$$

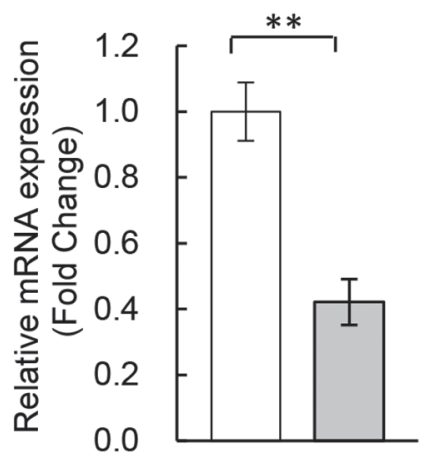

amimic-NC amimic
TNFa (ELISA)

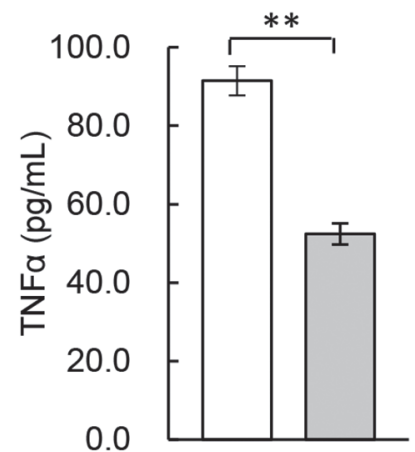

口NC_mimic $\square$ mimic
B TNF $\alpha$ (qRT-PCR)

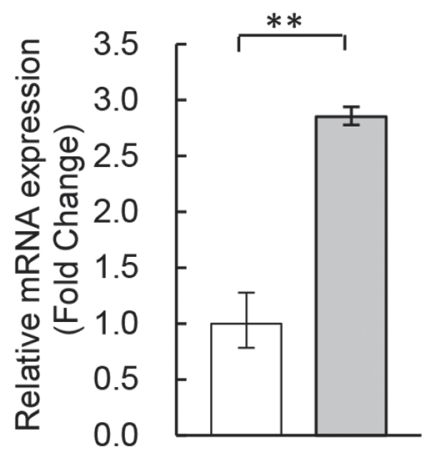

$\square$ NC_inhibitor $\square$ Inhibitor
TNFa (ELISA)

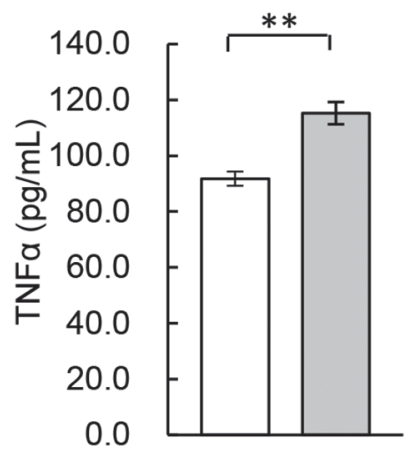

$\square$ NC_inhibitor $\square$ Inhibitor

C

IL-6 (qPCR)

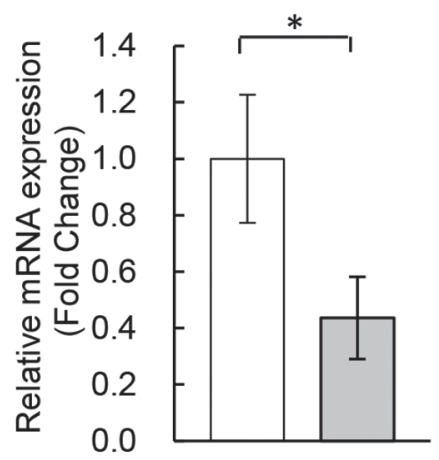

$\square$ mimic-NC $\square$ mimic
E

IL-8 (qPCR)

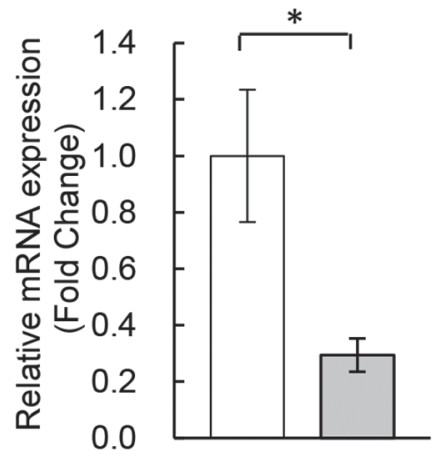

$\square$ mimic-NC $\quad \square$ mimic
IL-6 (ELISA)

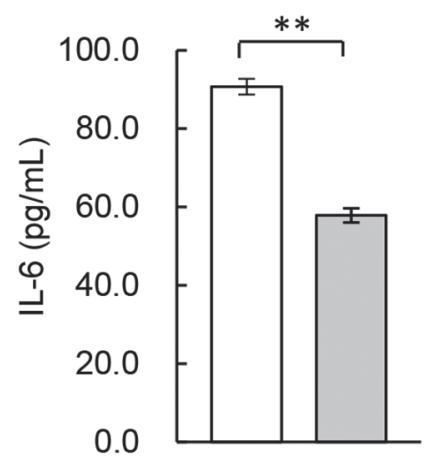

$\square$ NC_mimic $\square$ mimic
D

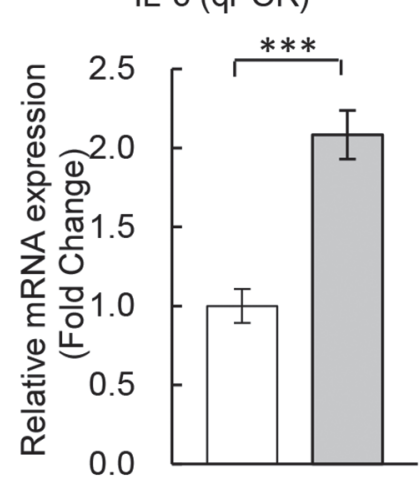

$\square$ NC_inhibitor $\square$ Inhibitor
IL-6 (ELISA)

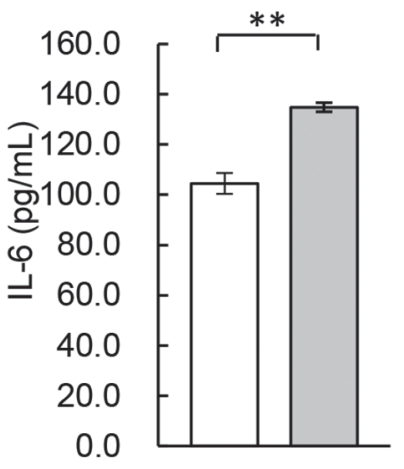

$\square$ NC_inhibitor $\square$ Inhibitor
F

IL-8 (ELISA)

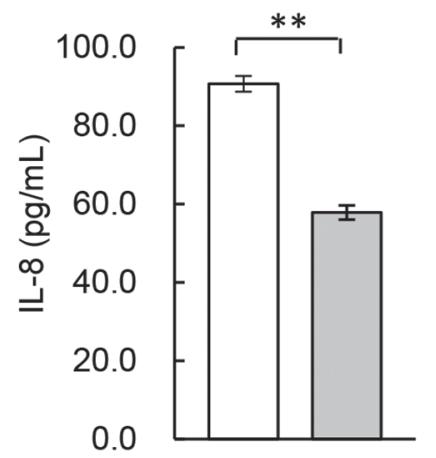

$\square$ NC_mimic $\square$ mimic
IL-8 (qPCR)

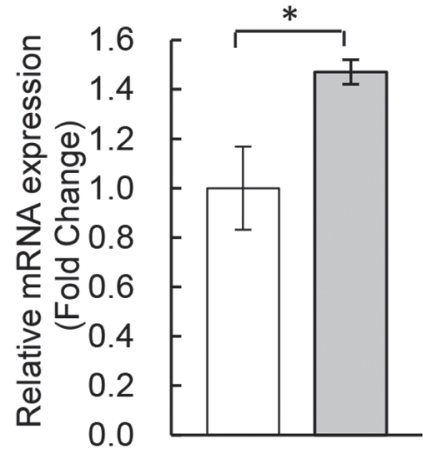

$\square$ NC_inhibitor $\square$ Inhibitor

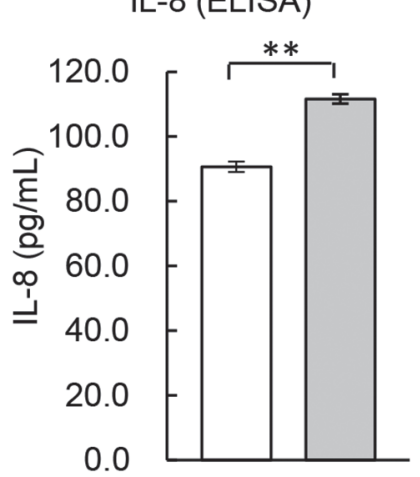

$\square$ NC inhibitor alnhibitor

Figure 4. Bovine miR-146a (bta-miR-146a) suppresses the production of tumor necrosis factor (TNF) $\alpha$, IL-6, and IL-8 in bovine mammary epithelial cells (bMEC). The bMEC were transfected with bta-miR-146a mimic or scrambled control mimic (NC_mimic); bta-miR-146a inhibitor or scrambled control inhibitor (NC_inhibitor). Twenty-four hours posttransfection, bMEC were challenged with LPS for $3 \mathrm{~h}$. Cells were then collected to measure mRNA expression and production of TNF $\alpha$ (A, B), IL-6 (C, D), and IL-8 (E, F). Levels of mRNA expression and production of inflammatory cytokines were determined by quantitative real-time PCR (qPCR) and ELISA, respectively. Data are shown as mean \pm SEM of 3 independent experiments. ${ }^{*} P<0.05,{ }^{* *} P<0.01,{ }^{* * *} P<0.001$. 
the expression of bta-miR-146a in the bovine mammary gland might be affected by multiple factors, such as bacterial type, bacterial dose, and infection time. Taken together, this suggests that the molecular mechanism of bta-miR-146a in bovine mastitis is highly complex and tightly regulated.

Regulation of gene expression by miRNA occurs through specific binding to the target gene that contains the complementary nucleotide sequence. It has been demonstrated that miR-146a inhibits the expression of IRAK1 and TRAF6 in humans and mice by directly targeting their $3^{\prime}$ UTR (Taganov et al., 2006; Dai et al., 2016). TRAF6 and IRAK1 are key adapter molecules in the TLR/IL-1 receptor signaling pathway, resulting in the mediation of NF- $\kappa \mathrm{B}$ signaling pathway activation in mammals. Notably, the sequences of miR146a, TRAF6, and IRAK1 genes between bovine and human are highly homologous, but not identical (Figure $1 \mathrm{~A}$ and $2 \mathrm{~A}$ ). Although miR-146a has been recognized to function in regulation expression of the TRAF 6 and IRAK1 genes in human and mouse (Taganov et al., 2006; Dai et al., 2016), the mechanistic role of btamiR-146a underlying bovine mammary inflammation remains unclear. In this study, we predicted multiple putative bta-miR-146a binding sites within IRAK1 and TRAF6, and performed $3^{\prime}$ UTR luciferase reporter assays. Our results indicated that bta-miR-146a directly targets the TRAF6 gene, a well-known mediator for $\mathrm{NF}-\kappa \mathrm{B}$ activation. However, we did not find any evidence indicating that bta-miR-146a targets the IRAKI gene. Furthermore, our data indicated that bta-miR146a mediated suppression of TRAF6 expression inhibits NF-kB activity. This finding is in agreement with previous studies in humans and mice (Taganov et al., 2006; Sha et al., 2013; Gao et al., 2015; Park et al., 2015). In the case of bta-miR-146b, we were only able to demonstrate that bta-miR-146b does not directly target the TRAF6 and IRAK1 genes. Further screening is required to elucidate target genes against which btamiR-146b acts.

The TRAF6 functions as an adaptor molecule in the NF- $\kappa \mathrm{B}$ pathway, and plays a crucial role in the initiation of the inflammatory response. This induc-

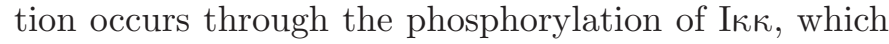
results in activation of NF- $\kappa \mathrm{B}$, an important transcription factor that regulates expression and secretion of inflammatory cytokines (Zhang and Ghosh, 2001; Griesbeck-Zilch et al., 2008; Fu et al., 2013). Interestingly, activated NF- $\kappa \mathrm{B}$ increases miR-146a expression by binding its promoter (Taganov et al., 2006). Once induced by NF- $\kappa \mathrm{B}$, increased expression of bta-miR$146 \mathrm{a}$ prevents the production of TRAF6 and NF- $\kappa \mathrm{B}$. Thus, we propose that expression of bta-miR-146a and

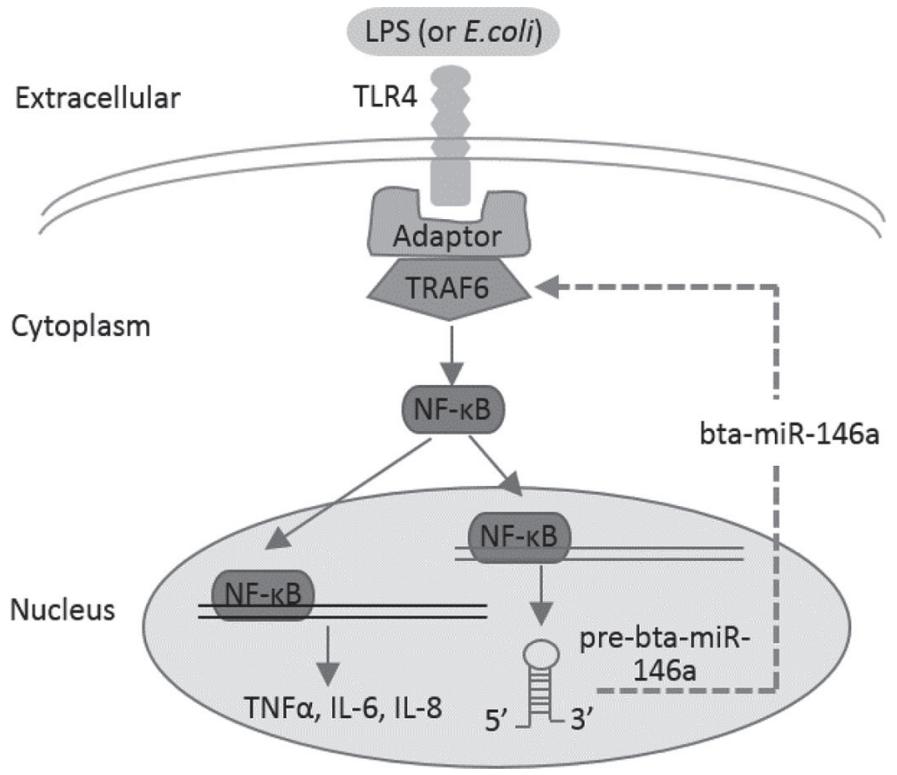

Figure 5. Schematic summary of the roles of bovine miR-146a (bta-miR-146a) in bovine mammary epithelial cells (bMEC). In response to LPS or Escherichia coli in bMEC, the toll-like receptor (TLR) 4 signaling pathway is activated, as is the tumor-necrosis factor receptor-associated factor 6 (TRAF6), which leads to the activation

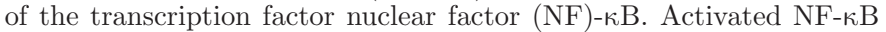
subsequently results in transcription of tumor necrosis factor (TNF) $\alpha$, IL-6, and IL-8, as well as bta-miR-146a. The substantial increase in bta-miR-146a expression downregulates the expression of TRAF6 in a negative-feedback cycle, leading to a suppression of TNF $\alpha$, IL-6, and IL-8.

its targeted degradation of TRAF6 mRNA functions as a negative feedback loop to control the expression of NF- $\mathrm{B}$, which is necessary for expression of inflammatory cytokines IL-6, TNFo, and IL-8 (Figure 5).

Once the pathogen enters the udder, bMEC respond to bacterial invasion and play an important role in the initiation of the antibacterial response (Rainard and Riollet, 2003). It has been demonstrated that heatinactivated $E$. coli or LPS activates NF- $\kappa$ B via the TLR4/NF- $\kappa B$ signaling pathway. In response to activation of the TLR signaling pathway, NF- $\kappa \mathrm{B}$ binding to the promoters of inflammatory cytokine genes results in increased expression of the cytokines TNFo, IL-6, IL-1, and IL-8 in bMEC at both mRNA and protein levels within 1 to $3 \mathrm{~h}$ of activation (Fu et al., 2013; Liu et al., 2014; Wang et al., 2016a). The TNFa and IL-6 are major pro-inflammatory cytokines that mediate the inflammatory response, especially IL-6, which plays an important role in the acute-phase response of inflammation (Le and Vilcek, 1989; Fu et al., 2013). Interleukin-8 is an important chemokine that recruits neutrophils and T-lymphocytes to the infection site, and is also involved in the activation of neutrophils 
(Harada et al., 1994; Strandberg et al., 2005). To clarify whether bta-miR-146a regulates the expression of bovine inflammatory cytokines, we used LPS-stimulated bMEC as a model for the process of inflammation. Our results demonstrated that bta-miR-146a can inhibit mRNA expression and secretion of TNF $\alpha$, IL-6, and IL8. The data presented here are indeed consistent with several previous reports in humans and mice (Li et al., 2013; Meisgen et al., 2014; Park et al., 2015).

In conclusion, we observed that bta-miR-146a downregulates TRAF6 and $N F-\kappa \mathrm{B}$ expression posttranscriptionally by directly targeting the $3^{\prime}$ UTR of TRAF6, consequently suppressing the production of the inflammatory mediators TNF $\alpha$, IL-6, and IL-8. This finding suggests that bta-miR-146a acts as a negative feedback regulator of inflammation through the downregulation of the TLR4/TRAF6/NF- $\kappa \mathrm{B}$ pathway. This study presents a regulatory mechanism of bta-miR-146a on immune responses of bovine mammary infection, and may also provide a potential therapeutic target for mastitis.

\section{ACKNOWLEDGMENTS}

This work was supported by grants from the National Natural Science Foundation of China (31301943, 31501935), National Science-Technology Support Plan Projects (2012BAD12B07), China Postdoctoral Science Foundation funded project (2014M562466), and the Construct Program of the Key Discipline in Hunan province (China).

\section{REFERENCES}

Bougarn, S., P. Cunha, F. B. Gilbert, F. Meurens, and P. Rainard. 2011. Technical note: Validation of candidate reference genes for normalization of quantitative PCR in bovine mammary epithelial cells responding to inflammatory stimuli. J. Dairy Sci. 94:24252430.

Bustin, S. A., V. Benes, J. A. Garson, J. Hellemans, J. Huggett, M. Kubista, R. Mueller, T. Nolan, M. W. Pfaffl, G. L. Shipley, J. Vandesompele, and C. T. Wittwer. 2009. The MIQE guidelines: Minimum information for publication of quantitative real-time PCR experiments. Clin. Chem. 55:611-622.

Dai, Y., P. Jia, Y. Fang, H. Liu, X. Jiao, J. C. He, and X. Ding. 2016. miR-146a is essential for lipopolysaccharide (LPS)-induced crosstolerance against kidney ischemia/reperfusion injury in mice. Sci. Rep. 6:27091.

Fu, Y., E. Zhou, Z. Liu, F. Li, D. Liang, B. Liu, X. Song, F. Zhao, X. Fen, D. Li, Y. Cao, X. Zhang, N. Zhang, and Z. Yang. 2013. Staphylococcus aureus and Escherichia coli elicit different innate immune responses from bovine mammary epithelial cells. Vet. Immunol. Immunopathol. 155:245-252.

Gao, M., X. Wang, X. Zhang, T. Ha, H. Ma, L. Liu, J. H. Kalbfleisch, X. Gao, R. L. Kao, D. L. Williams, and C. Li. 2015. Attenuation of cardiac dysfunction in polymicrobial sepsis by microRNA-146a is mediated via targeting of IRAK1 and TRAF6 expression. J. Immunol. 195:672-682.

Gilbert, F. B., P. Cunha, K. Jensen, E. J. Glass, G. Foucras, C. Robert-Granie, R. Rupp, and P. Rainard. 2013. Differential response of bovine mammary epithelial cells to Staphylococcus aureus or Escherichia coli agonists of the innate immune system. Vet. Res. 44:40.

Griesbeck-Zilch, B., H. H. Meyer, C. H. Kuhn, M. Schwerin, and O. Wellnitz. 2008. Staphylococcus aureus and Escherichia coli cause deviating expression profiles of cytokines and lactoferrin messenger ribonucleic acid in mammary epithelial cells. J. Dairy Sci. 91:2215-2224.

Gunther, J., M. Koy, A. Berthold, H. J. Schuberth, and H. M. Seyfert. 2016. Comparison of the pathogen species-specific immune response in udder derived cell types and their models. Vet. Res. (Faisalabad) 47:22.

Harada, A., N. Sekido, T. Akahoshi, T. Wada, N. Mukaida, and K. Matsushima. 1994. Essential involvement of interleukin-8 (IL-8) in acute inflammation. J. Leukoc. Biol. 56:559-564.

Huang, Y. Q., K. Morimoto, K. Hosoda, Y. Yoshimura, and N. Isobe. 2012. Differential immunolocalization between lingual antimicrobial peptide and lactoferrin in mammary gland of dairy cows. Vet. Immunol. Immunopathol. 145:499-504.

Jiang, W., L. Kong, Q. Ni, Y. Lu, W. Ding, G. Liu, L. Pu, W. Tang, and L. Kong. 2014. miR-146a ameliorates liver ischemia/reperfusion injury by suppressing IRAK1 and TRAF6. PLoS One 9:e101530.

Lawless, N., P. Vegh, C. Oâ Farrelly, and D. J. Lynn. 2014. The role of microRNAs in bovine infection and immunity. Front. Immunol. 5:611.

Le, J. M., and J. Vilcek. 1989. Interleukin 6: A multifunctional cytokine regulating immune reactions and the acute phase protein response. Lab. Invest. 61:588-602.

Li, S., Y. Yue, W. Xu, and S. Xiong. 2013. MicroRNA-146a represses mycobacteria-induced inflammatory response and facilitates bacterial replication via targeting IRAK-1 and TRAF-6. PLoS One 8:e81438

Liu, M., S. Song, H. Li, X. Jiang, P. Yin, C. Wan, X. Liu, F. Liu, and J. Xu. 2014. The protective effect of caffeic acid against inflammation injury of primary bovine mammary epithelial cells induced by lipopolysaccharide. J. Dairy Sci. 97:2856-2865.

Livak, K. J., and T. D. Schmittgen. 2001. Analysis of relative gene expression data using real-time quantitative $\mathrm{PCR}$ and the $2-\Delta \Delta \mathrm{CT}$ method. Methods 25:402-408.

Meisgen, F., L. N. Xu, A. Wang, B. Rethi, C. Bouez, M. Zuccolo, A. Gueniche, M. Stahle, E. Sonkoly, L. Breton, and A. Pivarcsi. 2014. MiR-146a negatively regulates TLR2-induced inflammatory responses in keratinocytes. J. Invest. Dermatol. 134:1931-1940.

Naeem, A., K. Zhong, S. J. Moisa, J. K. Drackley, K. M. Moyes, and J. J. Loor. 2012. Bioinformatics analysis of microRNA and putative target genes in bovine mammary tissue infected with Streptococcus uberis. J. Dairy Sci. 95:6397-6408.

O'Connell, R. M., D. S. Rao, A. A. Chaudhuri, and D. Baltimore. 2010. Physiological and pathological roles for microRNAs in the immune system. Nat. Rev. Immunol. 10:111-122.

Park, H., X. Huang, C. Lu, M. S. Cairo, and X. Zhou. 2015. MicroRNA-146a and MicroRNA-146b regulate human dendritic cell apoptosis and cytokine production by targeting TRAF6 and IRAK1 proteins. J. Biol. Chem. 290:2831-2841.

Rainard, P., and C. Riollet. 2003. Mobilization of neutrophils and defense of the bovine mammary gland. Reprod. Nutr. Dev. 43:439457.

Rainard, P., and C. Riollet. 2006. Innate immunity of the bovine mammary gland. Vet. Res. 37:369-400.

Seegers, H., C. Fourichon, and F. O. Beaudeau. 2003. Production effects related to mastitis and mastitis economics in dairy cattle herds. Vet. Res. 34:475-491.

Sha, M., J. Ye, L. Zhang, Z. Luan, and Y. Chen. 2013. Celastrol induces apoptosis of gastric cancer cells by miR-146a inhibition of NF- $\kappa B$ activity. Cancer Cell Int. 13:50.

Sordillo, L. M., K. Shafer-Weaver, and D. DeRosa. 1997. Immunobiology of the mammary gland. J. Dairy Sci. 80:1851-1865.

Strandberg, Y., C. Gray, T. Vuocolo, L. Donaldson, M. Broadway, and R. Tellam. 2005. Lipopolysaccharide and lipoteichoic acid induce different innate immune responses in bovine mammary epithelial cells. Cytokine 31:72-86. 
Taganov, K. D., M. P. Boldin, K. J. Chang, and D. Baltimore. 2006. NF-kappaB-dependent induction of microRNA miR-146, an inhibitor targeted to signaling proteins of innate immune responses. Proc. Natl. Acad. Sci. USA 103:12481-12486.

Vandesompele, J., K. De Preter, F. Pattyn, B. Poppe, N. Van Roy, A. De Paepe, and F. Speleman. 2002. Accurate normalization of real-time quantitative RT-PCR data by geometric averaging of multiple internal control genes. Genome Biol. 3:H0034.

Wang, J., C. Guo, Z. Wei, X. He, J. Kou, E. Zhou, Z. Yang, and Y. Fu. 2016a. Morin suppresses inflammatory cytokine expression by downregulation of nuclear factor- $\kappa \mathrm{B}$ and mitogen-activated protein kinase (MAPK) signaling pathways in lipopolysaccharidestimulated primary bovine mammary epithelial cells. J. Dairy Sci. 99:3016-3022

Wang, X. P., Z. M. Luoreng, L. S. Zan, S. H. A. Raza, F. Li, N. Li, and S. Liu. 2016b. Expression patterns of miR-146a and miR-146b in mastitis infected dairy cattle. Mol. Cell. Probes 30:342-344.
Wellnitz, O., and R. M. Bruckmaier. 2012. The innate immune response of the bovine mammary gland to bacterial infection. Vet. J. 192:148-152.

Wellnitz, O., and D. E. Kerr. 2004. Cryopreserved bovine mammary cells to model epithelial response to infection. Vet. Immunol. Immunopathol. 101:191-202.

Zeng, Z., H. Gong, Y. Li, K. Jie, C. Ding, Q. Shao, F. Liu, Y. Zhan, C. Nie, W. Zhu, and K. Qian. 2013. Upregulation of miR-146a contributes to the suppression of inflammatory responses in LPSinduced acute lung injury. Exp. Lung Res. 39:275-282.

Zhang, G., and S. Ghosh. 2001. Toll-like receptor-mediated NF-kap$\mathrm{paB}$ activation: A phylogenetically conserved paradigm in innate immunity. J. Clin. Invest. 107:13-19. 